\title{
NEOTIPIFICACIÓN Y RECONOCIMIENTO DE POLIANTHES GEMINIFLORA (LEX.) ROSE (AGAVACEAE)
}

\author{
Eloy Solano ${ }^{1,3}$ y Abisaí García-Mendoza ${ }^{2}$
}

${ }^{1}$ Universidad Nacional Autónoma de México, Unidad de Investigación en Sistemática Vegetal y Suelo, Herbario FEZA, Facultad de Estudios Superiores Zaragoza, Apdo. postal 9-020, Iztapalapa, 09230 México, D.F., México. ${ }^{2}$ Universidad Nacional Autónoma de México, Jardín Botánico, Instituto de Biología, Apdo. postal 70-614, Coyoacán, 04360 México, D.F., México. ${ }^{3}$ Autor para la correspondencia: solanoec@unam.mx

\section{RESUMEN}

Se designa el neotipo y se presenta una descripción detallada de Polianthes geminiflora (Lex.) Rose (Agavaceae). Esta especie fue descrita por Juan José Martínez de Lexarza como Bravoa geminiflora en Novorum vegetabilium descriptiones en 1824. Lexarza indicó que la planta fue recolectada en los montes de Valladolid, hoy Morelia, Michoacán. En 1903, J. N. Rose transfirió la especie al género Polianthes. Polianthes geminiflora tiene una distribución geográfica amplia y exhibe una gran variabilidad morfológica. Se han reconocido tres variedades, $P$. geminiflora var. clivicola, $P$. geminiflora var. geminiflora y $P$. geminiflora var. pueblensis. Además se presenta una clave dicotómica para la determinación de los taxa y un listado de los ejemplares de herbario consultados.

Palabras clave: Agavaceae, Bravoa, neotipo, Polianthes.

\section{ABSTRACT}

Polianthes geminiflora (Lex.) Rose (Agavaceae) is neotypified and its detailed description is presented. This species was described by Juan José Martínez de Lexarza as Bravoa geminiflora and published in Novorum vegetabilium descriptiones in 1824 . According to Lexarza, the plant was collected in the mountains of Valladolid, nowadays Morelia, Michoacán. In 1903 J. N. Rose transferred Bravoa geminiflora to P. geminiflora. Polianthes geminiflora has the widest geographical distribution and the highest morphological variation 
of the genus. Within $P$. geminiflora three infraspecific taxa have been recognized, $P$. geminiflora var. clivicola, $P$. geminiflora var. geminiflora and $P$. geminiflora var. pueblensis. Furthermore, we include a key to distinguish varieties and a list of herbarium specimens consulted.

Key words: Agavaceae, Bravoa, neotype, Polianthes.

\section{INTRODUCCIÓN}

Linneaus en 1753 dio a conocer el género Polianthes y su tipo es $P$. tuberosa. Lo clasificó en la clase VI Hexandria y orden I Monogynia, aunque con anterioridad dicho autor en Hortus Cliffortianus (1738), había utilizado el nombre Polianthes floribus alternis para referirse a estas plantas. Años más tarde, Pablo de La Llave y Juan José Martínez de Lexarza (1824) publicaron Novorum vegetabilium descriptiones, Fasciculus $I$, en donde propusieron 13 géneros y 40 especies nuevas para la ciencia. La mayoría de ellas fueron recolectadas en los alrededores de San José del Corral, Córdoba, Veracruz y en las áreas cercanas a Valladolid, hoy Morelia, Michoacán. Lexarza (1824) propuso el género Bravoa con una especie $B$. geminiflora.

Rose (1903) recalcó que no existen caracteres o grupo de ellos que justifiquen su separación y transfirió Bravoa geminiflora a Polianthes geminiflora, ampliando la descripción con base en ejemplares cultivados en un jardín de Londres y otros recolectados por él mismo en el Estado de México.

Solano (2000) indicó que Bravoa y Polianthes pueden separarse con base en caracteres morfológicos. En Bravoa la inflorescencia es racemosa y el perianto es de color rojo, anaranjado, anaranjado-verdoso, rosado con rayas blancas, o rara vez blanco. Las flores son tubulares, colgantes o depresas, los filamentos están insertos hasta $19 \mathrm{~mm}$ por arriba del ápice del ovario y las flores no tienen fragancia. Polianthes tiene flores agrupadas en espigas, el perianto es hipocrateriforme, blanco, rosado y hasta rojo con la edad. Las flores son ascendentes, difusas o divaricadas, los filamentos se insertan por debajo de los lóbulos del perianto y las flores son fragantes. Solano (2000) concordó que estas diferencias no son suficientes para considerarlos como géneros diferentes y los separó en dos subgéneros, Bravoa y Polianthes.

Según Stafleu y Cowan (1979), el material herborizado por Lexarza no se ha localizado y algunos de los especímenes correspondientes a de La Llave se encuentran en Ginebra (G-DC), pero Espejo-Serna et al. (1993) refieren que el Dr. Spichiger 
les comunicó que el único ejemplar tipo designado por de La Llave depositado en ese herbario es el de Alamania punicea Lex.

En los siguientes párrafos se presentan las descripciones originales de Bravoa, Bravoa geminiflora y Polianthes geminiflora. También se hace una descripción morfológica detallada de $P$. geminiflora y se designa el neotipo.

Bravoa Lex., in La Llave \& Lex. Nov. Veg. Descr. 1: 6. 1824.

Hexandria monoginia. Flores gemini spathacei. Perigonium infundibuliforme, ore amplificato. Filamenta; sex fundo tubi inserta, longitudine perigonii. Ovarium; trigonum adhaerens. Capsula; oblonga trilocularis, trivalvis. Semina; numerosa reniformia.

B. geminiflora Lex., in La Llave \& Lex. Nov. Veg. Descr. 1: 6. 1824.

Radix; bulbus solidus piriformis, tuberculis inferioribus fasciculatis. Scapus; longissimus, teres, articulatus, erectus, apice coloratus. Folia; radicalia ensiformia, patenti-reflexa, basi punctis coccineis maculata. Flores; alterni, gemini, penduli, breviter pedunculati, spatha squamiformi suffulti. Perigonium; infundibuliforme, fere cilindricum, extus puniceum, intus lutescens ore amplificato, limbo sex-dentato, dentibus tribus alternatim minoribus, paulullum introflexis, omnibus obtusis brevissimis. Filamenta; tubi fundo inserta, filiformia longitudine perigonii, antheris versatilibus. Ovarium; oblongum obscure trigonum, adhaerens, stylo incrassato, filamentis longiore, stigmate triquetro. Capsula; oblonga trilocularis, trivalvis perigonio persistenti obtecta. Semina; plurima, reniformia, duplici serie in unoquoque loculamento ordinata.

Habitat in montibus Micciacanis, et prope Vallisoletum. Floret autumno. -Lex.

Polianthes geminiflora (Lex.) Rose, Contr. U. S. Natl. Herb. 8: 12.1903.

Bravoa coetocapnia Roem., Fam. Nat. Syn. Monogr. 4: 245. 1847.

Bravoa geminiflora Lex., in La Llave \& Lex. Nov. Veg. Descr. 1: 6. 1824.

Coetocapnia geminiflora Link \& Otto, Ic. Pl. Rar. Hort. Berol. t. 18. 35. 1828.

Zetocapnia geminiflora Link \& Otto, loc. cit. t.18. 35. 1828.

Bravoa graminiflora Hemsl., Biol. Centr.-Am. 3: 338. 1884.

Bulbs rather shallow, giving off several thickened roots; stem 4 to $7 \mathrm{dm}$. tall, perfectly glabrous throughout; basal leaves several, 3 to $5 \mathrm{dm}$. long, 10 to $13 \mathrm{~mm}$. 
broad; stem leaves 3 or 4 , becoming successively shorter; racemes 2 to $4 \mathrm{dm}$. long; flowers in a few (6 or more) distant pairs; pedicels slender, 6 to $10 \mathrm{~mm}$. long; perianth rather pale red or orange-colored, about $2 \mathrm{~cm}$. long, strongly bent downward near the base; lobes short and broad, rounded at tip and slightly puberulent just below the tip (Rose, 1903).

Specimens examined: "England: Cultivated specimens taken from garden in London. State of Mexico: Meadow swells, valley of Toluca, C. G. Pringle, August 19, 1892 (no. 4215), and also from low meadows at Flor Maria, July 28, 1890 (no. 3633); near Tultenango, J. N. Rose, July 13, 1901 (no. 5420).”

Type locality: "In montibus Micciacanis, et prope Vallisoletum," or, as Hooker translates (Vol. 79, plate 4741.) "mountains of Valladolid and Miciciacan." Valladolid (now Morelia) is a town of the State of Michoacan.

Rose (1903) consideró a los tallos modificados como bulbos. Estudios recientes llevadas a cabo por González (1998) y Solano (2000) refieren que estas plantas presentan dos tipos de tallos hipógeos, un cormo con yemas y raíces contráctiles, por encima de éste se desarrolla un bulbo conformado por las bases de las hojas. El tallo de 4 a $7 \mathrm{dm}$ de alto descrito por Rose, corresponde al pedúnculo floral, mientras que, el racimo de 2 a $4 \mathrm{dm}$ alude a la parte fértil del mismo. La descripción original es breve, aquí se presenta con más detalle, basada en caracteres vegetativos y reproductivos y se designa un neotipo ante la ausencia de material original.

Polianthes geminiflora (Lex.) Rose, Contr. U. S. Natl. Herb. 8: 12, f. 4. 1903. Bravoa geminiflora Lex., in La Llave \& Lex. Nov. Veg. Descr. 1: 6. 1824. Agave duplicata Thiede \& Eggli, Kakteen And. Sukk. 50(5): 111. 1999. TIPO: MÉXICO: Micciacanis (Michoacán): in montibus Micciacanis, et prope Vallisoletum, J. M. Lexarza, sin haberse encontrado. NEOTIPO aquí designado: MÉXICO, Michoacán, Morelia, $4 \mathrm{~km}$ al sur de Jesús del Monte, 20 julio 1986, J. Rzedowski 39338 (neotipo: ENCB, isoneotipo IEB). Figs. 1, 2.

Coetocapnia geminiflora Link \& Otto (Zetocapnia), Icon. Pl. Rar. Hort. Berol. 35, t. 18. 1828. Bravoa coetocapnia (Link \& Otto) M. Roemer, Fam. Nat. Syn. Monogr. 4: 245. 1847. TIPO: MÉXICO: In regno Mexicano ad rincon de Temascaltepec, F. Deppe s.n., s.f. (holotipo: t. 18). 
Solano y García-Mendoza: Neotipificación y reconocimiento de Polianthes geminiflora (Agavaceae)

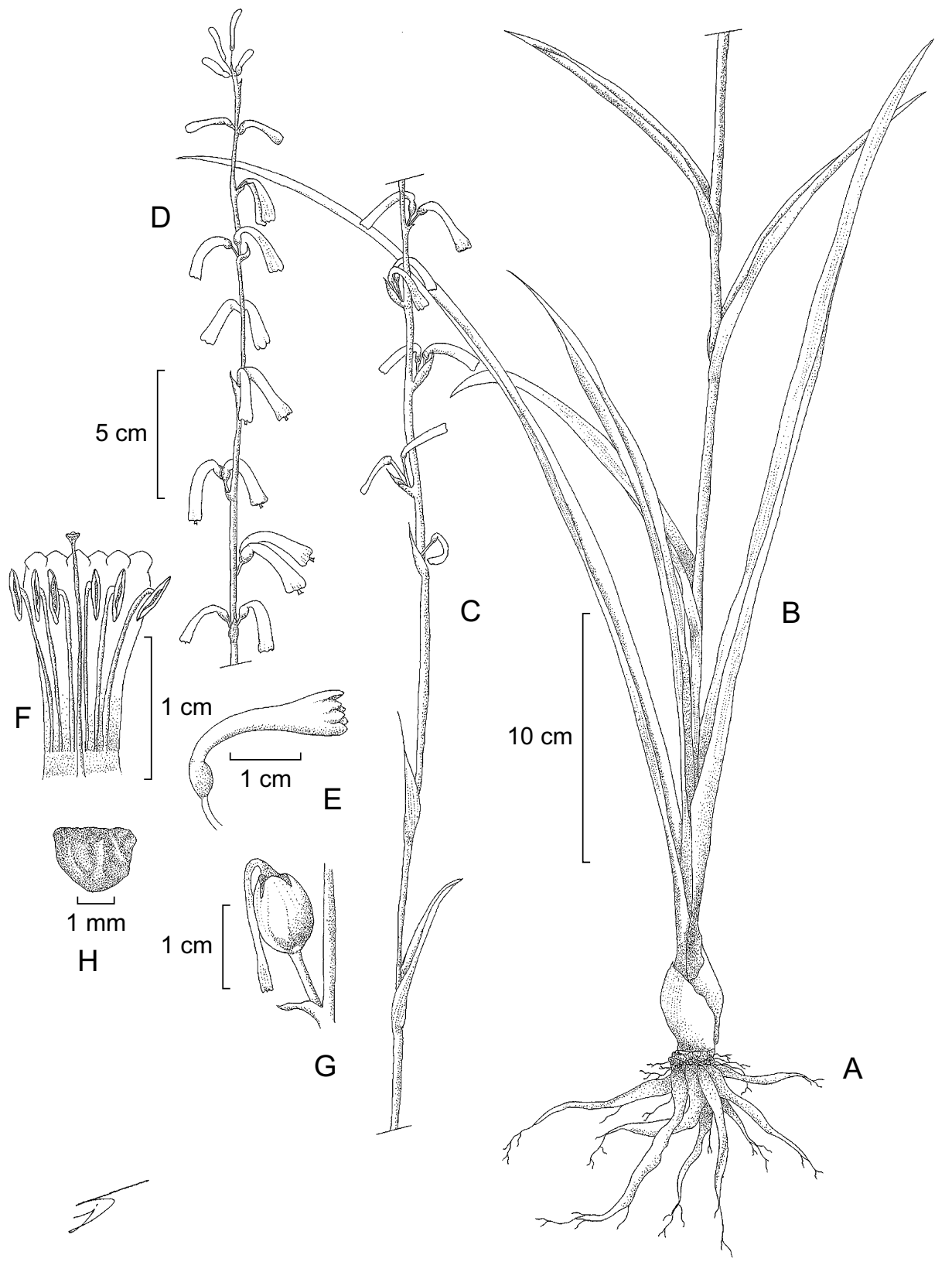

Fig. 1. Polianthes geminiflora. A. raíces contráctiles, cormo y bulbo; B. roseta y base del pedúnculo floral; $\mathrm{C}$ y D. porción media y distal de la inflorescencia; E. flor; F. flor disecada; G. fruto; H. semilla. Ilustración basada en el ejemplar E. Solano C. y C. Correa D. 1131 (FEZA, MEXU). 


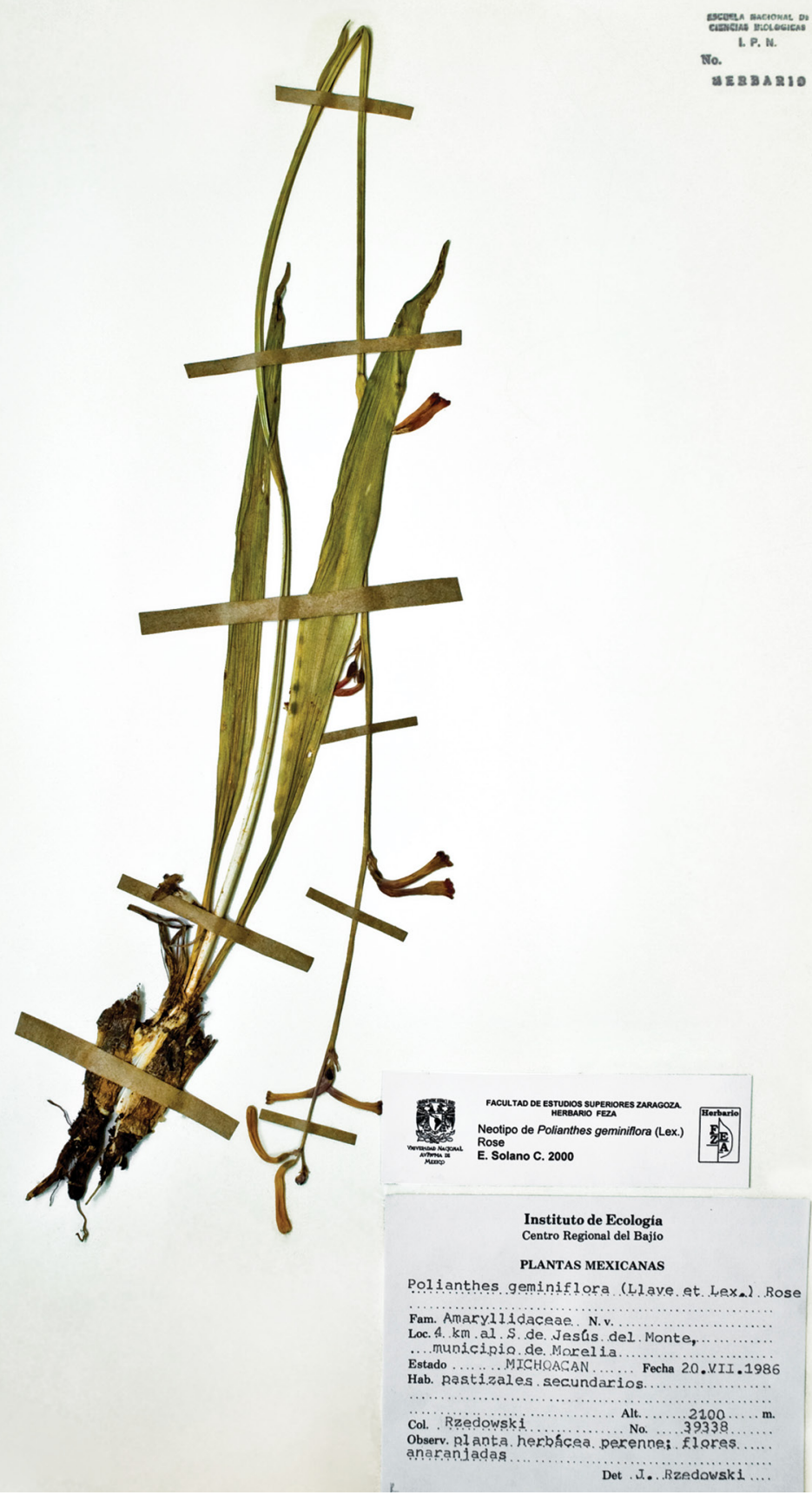

Fig. 2. Neotipo de Polianthes geminiflora (Lex.) Rose, J. Rzedowski 39338 (ENCB). 
Robynsia geminiflora Drapiez in Lem., Hort. Universel 2: 231. 1841. TIPO: MÉXICO: De una planta viva cultivada en Bruselas, descubierta en México por $H$. Galeotti s.n., s.f. (holotipo: t. 36).

Polianthes americana Sessé \& Mociño, Pl. Nov. Hisp. ed. 2. p. 50. 1893. TIPO: MÉXICO: In Sancti Angeli hortis prope Mexicum. Polyanthes tubulata Sessé \& Mociño, Fl. Mex. ed. 2. p. 88. 1894.

Hierbas perennes. Cormo 1.5-2.7 cm de largo, 1.0-2.0 cm de diámetro, cilíndrico, con raíces contráctiles carnosas y fibrosas. Bulbo 1.8-4.0 cm de largo, 1.3-3.0 cm de diámetro, ovoide, cubierto por las bases de las hojas secas. Hojas 2-6(-8) por roseta, alternas espiraladas, (8-)12-35(-50) cm de largo, 1-25(-37) $\mathrm{mm}$ de ancho, linear-lanceoladas a largamente atenuadas hacia el ápice, margen entero, denticulado, papiloso o eroso, ápice agudo; envés liso, base de las hojas con máculas purpúreas. Inflorescencia racemosa, laxa, densa hacia su porción distal, 35-100(-154) $\mathrm{cm}$ de largo, incluido el pedúnculo floral, con la base glabra, 5-15(-28) nudos florales; primera bráctea estéril, 4.5-31 cm de largo, 1-23(-28) $\mathrm{mm}$ de ancho, atenuada hacia el ápice, linear a lanceolada, ápice agudo, margen liso, papiloso, denticulado o eroso, las siguientes reduciéndose gradualmente en tamaño hacia la porción distal; primera bráctea floral 0.5-1.0(-2.0) $\mathrm{cm}$ de largo, ovado-lanceolada u ovada, algunas veces con el ápice obtuso, comúnmente acuminado o caudado, bracteolas dos, 1.5-3.0 $\mathrm{mm}$ de largo, lineares, lanceoladas a ovadas, ápice agudo a acuminado, escariosas. Flores pediceladas, pedicelos (0.3-)1.0-1.8 cm de largo, tubulares, geminadas, colgantes, rojas, anaranjadas o de color coral; boca del tubo regular a ligeramente oblicua, sin fragancia; tubo periantal 14-21 mm de largo, 1-3(-5) mm de ancho en la base de los lóbulos, lóbulos externos e internos mucho más cortos que el tubo, ligeramente desiguales, 2-3(-5) $\mathrm{mm}$ de largo, 1.0-2.5 $\mathrm{mm}$ de ancho, ovados, orbiculares, suborbiculares a transversalmente elípticos, erguidos, cuculados, ápice obtuso a redondeado, con un mechón de tricomas cortos de color blanco; estambres inclusos, filamentos filiformes, (5-)10-21(-24) $\mathrm{mm}$ de largo, insertados 2-5 $\mathrm{mm}$ por arriba del ápice del ovario, blancos; anteras 3-6 mm de largo, lineares, amarillas, versátiles, estilo 1.0-2.5 cm de largo, a veces tan largo como el tubo del perianto, filiforme, blanco; estigma trilobado. Fruto capsular, 0.7-1.7 cm de largo, 0.5-1.2 cm de ancho, globoso, coronado por restos del perianto. Semillas de 2-4(-5) $\mathrm{mm}$ de largo, 2-3 $\mathrm{mm}$ de ancho, semicirculares vistas de perfil, planas, dispuestas en dos hileras por lóculo, negras, brillantes u opacas. 
Distribución. Se distribuye desde Durango y Nayarit en el noroeste hasta Puebla y Guerrero en el sur (Fig. 3), en altitudes de 900 a 2460 m.

Polianthes geminiflora es la especie del género con mayor amplitud geográfica y ecológica, por consiguiente es muy diversa morfológicamente. Esta variabilidad se refleja en el tamaño de la planta, las hojas, las brácteas, la inflorescencia y el número de nudos fértiles. La variación en la forma y el tamaño del tubo del perianto, no es tan evidente y según McVaugh (1989) puede ser más aparente que real.

Con base en el tipo de margen de las hojas, McVaugh (1989) distinguió dos variedades: Polianthes geminiflora var. geminiflora y P. geminiflora var. clivicola. La primera de ellas tiene una amplia distribución geográfica en México, mientras

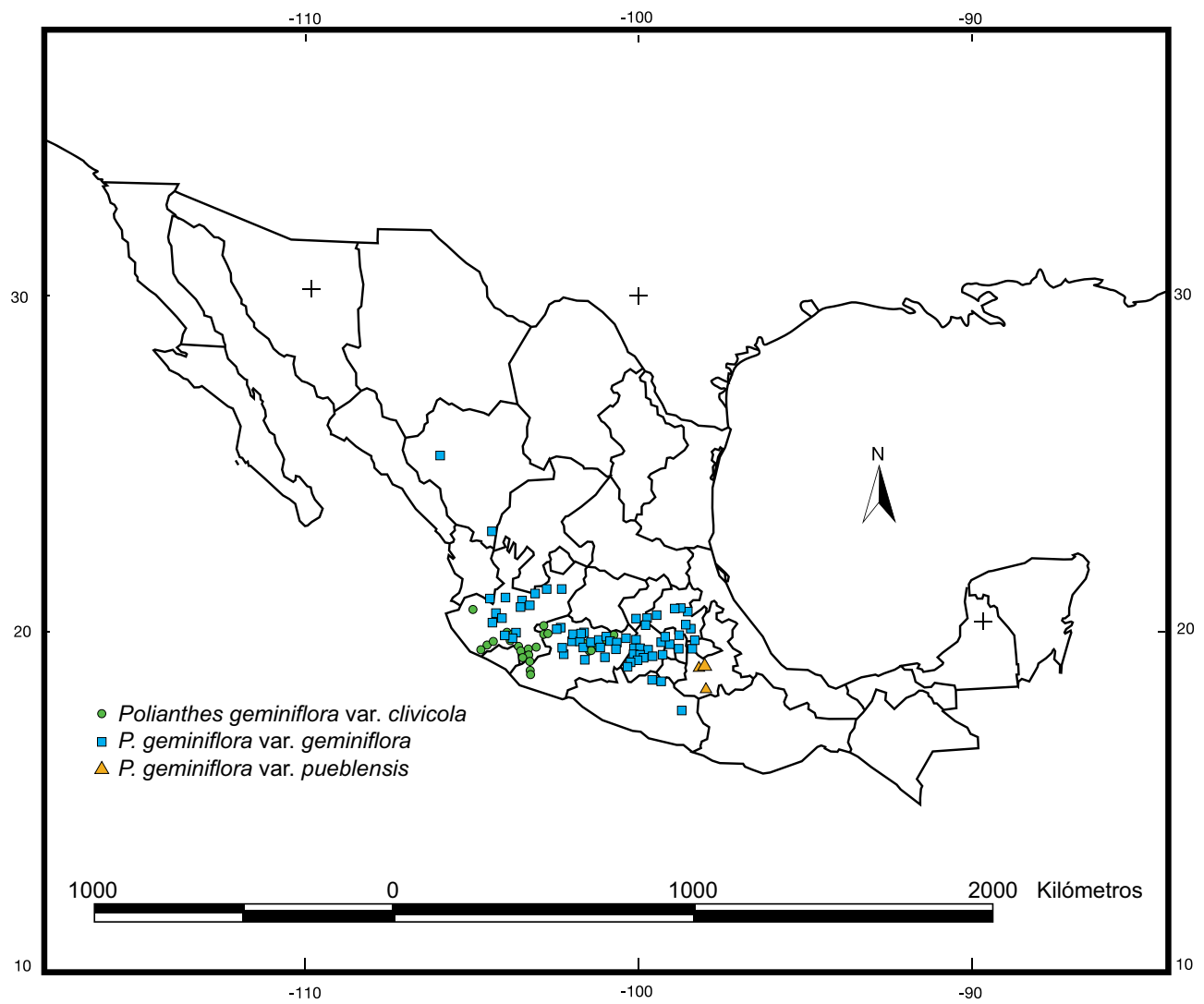

Fig. 3. Distribución geográfica de Polianthes geminiflora (Lex.) Rose. 
que la segunda es endémica del sur y oeste de Jalisco y Michoacán. García-Mendoza y Solano (2007), propusieron además a $P$. geminiflora var. pueblensis, propia del estado de Puebla. Estos taxa se separan de acuerdo con la siguiente clave:

1 Hojas con los márgenes enteros o papilosos, papilas en el envés, hojas 3-37 mm de ancho

2 Margen de las hojas translúcido, entero, hialino o escarioso, revoluto P. geminiflora var. clivicola

2 Margen de las hojas verrucoso-papiloso, frecuentemente eroso o irregularmente papiloso P. geminiflora var. geminiflora

1 Hojas con los con márgenes regularmente denticulados, papilosas en el envés, hojas 1-2 $\mathrm{mm}$ de ancho P geminiflora var. pueblensis

Polianthes geminiflora (Lex.) Rose var. clivicola McVaugh, Fl. Novo-Galiciana 15: 250, 1989. Agave duplicata Thiede \& Eggli ssp. clivicola (McVaugh) Thiede \& Eggli, Kakteen And. Sukk. 50(5): 111. 1999. TIPO: MÉXICO: Jalisco: 10 miles south of Autlán, 5 august 1949. R. L. Wilbur y C. R. Wilbur 2133 (holotipo: MICH!).

Hierbas perennes. Hojas 2-4(-8) por roseta, (15-)25-30(-50) cm de largo, (3-)15$25(-37) \mathrm{mm}$ de ancho, lineares, margen delgado, translúcido, hialino o escarioso, liso, generalmente muy angosto. Inflorescencia racemosa, (20-)45-100(-154) cm de largo; primera bráctea estéril, 4.5-15(-30) $\mathrm{cm}$ de largo, 4-28 $\mathrm{mm}$ de ancho, margen translúcido, entero, hialino o escarioso, revoluto. Flores con el tubo periantal de $14-20 \mathrm{~mm}$ de largo, 1-3 mm de ancho por debajo de los lóbulos, lóbulos internos y externos 2-4 $\mathrm{mm}$ de largo, 1.5-2.5 $\mathrm{mm}$ de ancho; filamentos 9-15 $\mathrm{mm}$ de largo, insertados 2-3(-5) $\mathrm{mm}$ por arriba del ápice del ovario, anteras $3.5-5.0 \mathrm{~mm}$ de largo. Fruto $0.7-1.0 \mathrm{~cm}$ de largo, $0.7-1.0 \mathrm{~cm}$ de ancho.

Fenología. Florece de junio a agosto y fructifica de septiembre a noviembre.

Hábitat y distribución. Se encuentra en áreas abiertas de bosque de encino, pino, pino-encino, encino-pino, pastizales y selva mediana subcaducifolia en ecotonía con bosque de encino. En suelos de color rojo, amarillo, negro o pardo oscuro; con textura arcillosa o arenosa, profundos, muy húmedos a veces con problemas de drenaje; en poblaciones muy localizadas y con pocos individuos, a veces forma grupos relativamente grandes. Se distribuye en Jalisco y Michoacán. En altitudes de 900 a $2200 \mathrm{~m}$. 
Nombre común. Coralillo (Michoacán).

Ejemplares examinados: JALIsco: Mpio. Autlán de Navarro: 30 julio 1989, $A$. Espejo \& A. R. López 3750 (UAMIZ); 18 to 20 miles southwest of Autlán, 23 julio 1951, H. Scott 10971 (MEXU, MICH). Mpio. Casimiro Castillo: cerro de microondas puerto los Mazos entre Autlán de Navarro y Casimiro Castillo, 22 agosto 1987, M. Cházaro \& P. Hernández 4946 (IEB, XAL). Mpio. Pihuamo: sobre la carretera 110 yendo de Tecalitlán hacia Pihuamo, entre los kilómetros 139-143, 11 agosto 1996, E. Solano \& C. Correa 866 (FEZA, MEXU); carretera 110 Pihuamo - Colima, entre los km 171 y 172, aproximadamente $1 \mathrm{~km}$ antes de llegar a Santa Cruz, $3 \mathrm{~km}$ al SW de Pihuamo, 11 agosto 1996, E. Solano \& C. Correa 867 (FEZA, MEXU). Mpio. Tamazula: cerca de Agua Hedionda, más o menos $45 \mathrm{~km}$ al E del aserradero sobre el camino a Manuel M. Diéguez, 26-27 octubre 1973, J. Rzedowski \& R. McVaugh 1126 (MICH); casi $13 \mathrm{~km}$ al E de Tamazula, sobre la brecha a San Juan de la Montaña, 19 octubre 1989, J. Villa et al. 98 (IEB). Mpio. Tapalpa: $10 \mathrm{~km}$ al S de Tapalpa camino a Venustiano Carranza, E. Lott et al. 400 (ENCB, XAL). Mpio. Tecalitlán: Barranca de San Juan de Dios, about $15 \mathrm{~km}$ east of Pihuamo, eastern slopes of Sierra de los Corales, 24 octubre 1963, J. V. A. Dieterle 3021 (MICH); Puente San Pedro, 5 miles SW of Tecalitlán, 9 agosto 1957, R. McVaugh 16016 (MEXU, MICH); ca. 10 km al E de Tecalitlán, sobre la brecha a la Nogalera y Jilotlán, 11 octubre 1989, J. Villa \& J. Chávez 94 (CHAPA). Sin municipio especificado: Cerro Santa María, 8-10 $\mathrm{km}$ southwest of Jiquilpan and ca. $5 \mathrm{~km}$ northeast of Quitapan, Jal., 8-9 agosto 1959, C. Feddema 224 (MICH); La Palma, 9 junio 1892, M. E. Jones 460 (MICH, US); al SE de la laguna de Chapala, 10 junio 1892, M. E. Jones 469 (MICH), $1.5 \mathrm{~km} \mathrm{~N}$ of Pihuamo, 31 julio 1960, H. Iltis et al. 629 (MEXU, MICH); Sierra del Halo, near a lumber road leaving the Colima highway 7 miles south-southwest Tecalitlán and extending south easterly toward San Isidro, 13 agosto 1957, R. McVaugh 16120 (IEB, MICH, NY); 48 miles from junction of bypass to Colima, 26 julio 1975, Wallace et al. 319 (ENCB). Michoacán: Mpio. Coalcomán: Filo de Salitre, 9 octubre 1938, G. B. Hinton et al. 12165 (GH, MICH, NY, RSA); S. Naranjillo, 13 julio 1939, G. B. Hinton 13921 (GH, NY); Puerto Zarzamora, east of Coalcomán, 3 agosto 1939, G. B. Hinton 15042 (GH, IEB, MICH, NY, RSA). Mpio. Villa Madero: Poruás, 13 noviembre 1987, H. Díaz 4984 (MEXU). Mpio. Zinapécuaro: Ucareo, 12 julio 1986, S. Zamudio 4115 (IEB, MEXU).

Polianthes geminiflora (Lex.) Rose var. geminiflora. Agave duplicata Thiede \& Eggli ssp. duplicata, Kakteen And. Sukk. 50(5): 111. 1999. 
Hierbas perennes. Hojas 2-4 por roseta, 13-25 cm de largo, (1.5-)5-15 mm de ancho, lineares, margen verrucoso-papiloso, frecuentemente eroso o irregularmente papiloso. Inflorescencia un racimo, (20-)40-90(-125) cm de largo; primera bráctea estéril, (6-)10-31 cm de largo, 2-10 mm de ancho, margen papiloso. Flores con el tubo periantal de 14-20 mm de largo, 1-3(-5) mm de ancho por debajo de los lóbulos; lóbulos exteriores e interiores, 2-4(-5) $\mathrm{mm}$ de largo, 1.5-2.5 $\mathrm{mm}$ de ancho; filamentos 5-15(24) mm de largo, insertados 2-3(-5) mm por arriba del ápice del ovario; anteras 3-6 mm de largo. Fruto 0.7-1.0(-1.7) $\mathrm{cm}$ de largo, 0.7-1.2 $\mathrm{cm}$ de ancho.

Fenología. Florece de junio a agosto y fructifica de septiembre a noviembre.

Hábitat y distribución. Crece en áreas abiertas de bosque de encino, pino, pino-encino, encino-pino y Juniperus; matorral xerófilo y pastizal. Se desarrolla sobre suelos profundos de color rojo, negro, pardo-oscuro, con textura arcillosa o arenosa, muy húmedos, a veces con problemas de drenaje. Las poblaciones pueden ser pequeñas o grandes. Se distribuye en el Distrito Federal, Durango, Estado de México, Hidalgo, Jalisco, Michoacán, Nayarit, Querétaro y Tlaxcala. En altitudes de 900 a $2460 \mathrm{~m}$.

Nombres comunes. Hierba de la pipa (Durango), coralillo, flor de María, perrito (Estado de México); calzoncito de San Miguel, charanguini en purépecha, coral, coralito, lirio (Michoacán).

Ejemplares examinados: Distrito Federal: Valle de México, agosto 1954, D. B. Gold 582 (MEXU); Sierra de Guadalupe, al N de la ciudad de México, agosto 1952, L. Paray 573 (ENCB); Sierra de Guadalupe, 11 agosto 1940, F. Miranda 584 (MEXU); Sierra de Guadalupe, Cerro Grande, $5 \mathrm{~km}$ al NNW de Cuautepec, 30 junio 1973, S. Moreno 249 (ENCB, MEXU); Sierra de Guadalupe, $5 \mathrm{~km}$ al NNW de Cuautepec, 29 agosto 1971, J. Rzedowski 28585 (MICH). Durango: Mpio. Mezquital: $19 \mathrm{~km}$ al SW de Charcos, camino a Santa María Ocotán, 5 octubre 1983, R. Fernández 1834 (ENCB). Mpio. Tepehuanes: Mesa de los Nayar, 14 septiembre 1989, O. Bravo 510 (CHAP). Estado de MÉXICo: Mpio. Aculco: Fondó, frente a la Escuela secundaria Gustavo Baz, 15 julio 1995, E. Solano et al. 796 (FEZA, MEXU). Mpio. Atizapán: $4 \mathrm{~km}$ al $\mathrm{N}$ de Atizapán, cerca de Madín al S de la Colmena, 21 agosto 1968, J. Rzedowski 26007 (MICH); Cerro del Tigre al NW de Atizapán, 4 agosto 1974, J. Rzedowski 31999 (CHAPA, ENCB, MEXU). Mpio. Coyotepec: ladera W de la sierra de Alcaparrosa, cerca de la torre de microondas, 7 septiembre 1980, R. Galván 677 
(ENCB, IEB); parte alta de la sierra de Alcaparrosa, $5 \mathrm{~km}$ al $\mathrm{W}$ de Coyotepec, 16 septiembre 1981, R. Galván 1018 (ENCB); parte alta de la sierra de Alcaparrosa, 5 km al W de Coyotepec, 28 agosto 1983, R. Galván 1419 (ENCB); 8 km al NW de Tepotzotlán, $2 \mathrm{~km}$ adelante de la estación retransmisora, 14 agosto 1981, J. García 1468 (CHAPA); ladera W de la Sierra Colorada, a un costado de la estación retransmisora, 1 agosto 1997, E. Solano et al. 1103 (FEZA, MEXU). Mpio. Nicolás Romero: $1 \mathrm{~km}$ al W de Magú, 27 junio 1963, J. Rzedowski 16831 (ENCB, MICH); 2 km al NW de Magú, 1 agosto 1997, E. Solano et al. 1100 (FEZA, MEXU); $1 \mathrm{~km}$ al NE de Miranda, sobre la cima de la loma Xalango, $3 \mathrm{~km}$ al SE de Cahuacán, 1 agosto 1997, E. Solano et al. 1102 (FEZA, MEXU). Mpio. Otumba: 18 julio 1981, M. Castilla \& J. D. Tejero 1366 (IZTA). Mpio. San Felipe del Progreso: carretera Villa Victoria - El Oro, $14 \mathrm{~km}$ al N de la Palizada, alrededores del vivero municipal, 23 agosto 1997, E. Solano \& C. Correa 1131 (FEZA, MEXU); carretera Villa Victoria - El Oro, 4 km al N de la Palizada, San Miguel Agua Bendita, 23 agosto 1997, E. Solano \& C. Correa 1132 (FEZA, MEXU). Mpio. San Martín de las Pirámides: las Tetillas del Cerro Gordo, 18 julio 1981, M. Castilla \& J. D. Tejero 1366b (ENCB). Mpio Tejupilco: sierra de Nanchititla; 18 agosto 1979, E. Guizar 586 (CHAP, MEXU). Mpio. Temascaltepec: Mina de Agua, 24 agosto 1932, G. B. Hinton 1412 (NY, US). Nanchititla, 14 agosto 1933, G. B. Hinton 4527 (NY, RSA, US); Mina de Agua, 24 agosto 1933, G. B. Hinton 4456 (GH, NY, RSA). Mpio. Tepotzotlán: sierra de Alcaparrosa, 12 julio 1983, J. E. N. Reynoso 907 (IZTA); $5 \mathrm{~km}$ al NW de Tepotzotlán, 29 julio 1971, J. Rzedowski 28302 (ENCB, MICH). Mpio. Texcoco: 8 km al E de Coatlinchán, 2 agosto 1981, R. Galván 954A (CHAP, ENCB, IEB, UAMIZ); $8 \mathrm{~km}$ al E de Coatlinchán, 20 julio 1967, J. Rzedowski 24071 (ENCB, MEXU, MICH); $8 \mathrm{~km}$ al E de Coatlinchán, 16 agosto 1977, J. Rzedowski 35150 (ENCB, MEXU); carretera Texcoco - Tlaxcala a la altura del km 41, 8 julio 1995, E. Solano et al. 789 (FEZA, MEXU); Coatlinchán, $10 \mathrm{~km}$ al SE de Coatlinchán, sobre el camino de terracería hacia el Cerro Quetzaltepec, 26 junio 1997, E. Solano \& C. Correa 1087 (FEZA, MEXU); Coatlinchán, $12 \mathrm{~km}$ al E de Coatlinchán, 26 junio 1997, E. Solano \& C. Correa 1089 (FEZA, MEXU); Coatlinchán, $13 \mathrm{~km}$ al SE de Coatlinchán, falda W del Cerro Quetzaltepec, E. Solano \& C. Correa 1090 (FEZA, MEXU); Tequesquinahuac, $1 \mathrm{~km}$ al E de Tequesquinahuac, camino de terracería a San Pablo Ixayoc, 27 junio 1997, E. Solano \& C. Correa 1091 (FEZA, MEXU). Mpio. Tlalnepantla: cerca de Tlalnepantla, 7 julio 1951, E. Matuda 21688 (MEXU). Mpio. Toluca: 20 miles West of Toluca, 12 julio 1940, C. L. Hitchcock \& L. R. Stanford 7123 (US). Mpio. Valle de Bravo: septiembre 1952, E. Lyonnet 954A (MEXU). Mpio. Villa Victoria: rancho Buena Vista, km 40 carretera Toluca - 
Zitacuaro, 24 julio 1989, A. R. López \& A. Espejo 876 (IEB, MEXU, UAMIZ); aproximadamente, $6 \mathrm{~km}$ al $\mathrm{N}$ de Villa Victoria, sobre la carretera Villa Victoria - El Oro, 23 agosto 1997, E. Solano \& C. Correa 1130 (FEZA); cerca de la presa, $6 \mathrm{~km}$ al SW de Villa Victoria, 6 julio 1985, J. C. Soto et al. s/n (MEXU); km 4 Villa Victoria - El Oro; 30-31 agosto 1986, J. D. Tejero \& M. Castilla 2545 (IZTA). Sin municipio especificado: 20 miles west of Toluca, 12 julio 1940, C. L. Hitchcock \& L. R. Stanford (US); km 165.5 carretera México - Morelia, septiembre 1983, G. Palomino et al. 72 (MEXU); km 19 México - Toluca al norte, 20 agosto 1940, I. Langman 2748 (MEXU); Cerro de Nanchititla, Distrito de Tejupilco, en la falda sur, 8 septiembre 1954, E. Matuda 31592 (MEXU); sin localidad, 28 julio 1890, C. G. Pringle 3633 (GH); Valley of Toluca, 19 agosto 1892, C. G. Pringle 4215 (GH, MEXU, NY, UC, US); Cerro de la Cruz, $5 \mathrm{~km}$ al NW de Tepotzotlán, 23 julio 1974, J. Rzedowski 31968 (ENCB, MEXU). Sin municipio especificado: Sierra de Guadalupe, falda norte, 17 junio 1951, E. Matuda 21356 (MEXU); Sierra de Guadalupe, falda norte, 22 julio 1951, E. Matuda 21530 (CHAP, ENCB, MEXU, UAMIZ); Estado de México, 11 agosto 1885, W. Schumann 1475 (US). GuERrero: Mpio. Taxco: sin localidad, 21 julio 1937, R. Q. Abbott 265 (ENCB, GH); between Casahuates and small reservoir at head of waterfall above town on mountains west of and above Taxco, 17 agosto 1948, H. E. Moore \& C. E. Wood 4575. Mpio. Tlapa: Aguatecocomolitic, $1 \mathrm{~km}$ al $\mathrm{N}$ de Chiepetepec, carretera Tlapa - Chilapa, $13 \mathrm{de}$ agosto de 1999, A. García M. et al. 6886 (MEXU). Hidalgo: Mpio. Ajacuba: Los Pechitos al sur del poblado de Ajacuba, vertiente $\mathrm{N}$ de la sierra del Monte Alto de Temoaya, ejido Ajacuba, 17 agosto 1990, I. Díaz et al. 889 (MEXU). Mpio. Huasca de Ocampo: camino a Zembo, $4 \mathrm{~km}$ al SSE de Huasca, 19 agosto 1989, M. Medina \& M. A. Barrios 3929 (MEXU); $3 \mathrm{~km}$ al SE de Huasca, 28 junio 1997, E. Solano \& C. Correa 1094 (FEZA, MEXU). Mpio. Huichapan: Presa Madero, $12 \mathrm{~km}$ al E de Huichapan, 24 junio 1980, R. Hernández \& R. Hernández 4556 (MEXU, XAL); 5 $\mathrm{km}$ al E de Jonacapa, $17 \mathrm{~km}$ al E de Huichapan, 25 junio 1980, $R$. Hernández \& $R$. Hernández 4588 (MEXU). Mpio. Zacualtipán: Piedra Blanca, 5 agosto 1995, J. Reyes et al. 3501 (FEZA); aproximadamente $20 \mathrm{~km}$ antes de llegar a Zacualtipán, viniendo de Atotonilco el Grande, 29 junio 1997, E. Solano \& C. Correa 1095 (FEZA, MEXU); aproximadamente $18 \mathrm{~km}$ antes de llegar a Zacualtipán, viniendo de Atotonilco el Grande, 29 junio 1997, E. Solano \& C. Correa 1096 (FEZA, MEXU); aproximadamente $2 \mathrm{~km}$ antes de llegar al ejido Atopixco, sobre la carretera Atotonilco el Grande - Zacualtipán, 29 junio 1997, E. Solano \& C. Correa 1098 (FEZA, MEXU). Sin municipio especificado: $5 \mathrm{~km}$ al E de Jonapan, $17 \mathrm{~km}$ al E de Huichapan, 25 junio 1980, R. Hernández \& R. Hernández 4588 (MEXU); near 
Buenavista, 4 agosto 1904, C. G. Pringle 13221 (GH, MICH); between Somoriel and Las Lajas, 5 agosto 1905, J. Rose et al. 9164 (GH, NY). JALISCO: Mpio. Ixtlahuacán: several miles south of Ixtlahuacán on México hwy 71, 21 julio 1957, R. M. Straw \& D. P. Gregory 996 (MICH). Mpio. San Cristóbal de la Barranca: $10 \mathrm{~km}$ al N de la desviación a San Cristóbal de la Barranca, carretera a Tesistán, antes de llegar al devisadero, 4 de agosto 1988, A. Flores et al. 1017 (IEB, MEXU); San Cristóbal de la Barranca, 16 agosto 1990, A. R. López \& A. Espejo 1347 (UAMIZ). Mpio. Tapalpa (Venustiano Carranza): $3 \mathrm{~km}$ al W de la Frontera, 10 julio 1990, J. J. Guerrero et al. 850 (IEB); potrero las Manzanillas, 6 agosto 1924, S. Walker 74 (NY). Mpio. Tequila: Cerro de Tequila, $\mathrm{km} 7$ de la terracería, 6 septiembre 1988, $S$. González s/n (MEXU). Mpio. Zapopan: km 14 por la carretera Zapopan San Cristóbal de la Barranca, 18 septiembre 1989, F. J. Santana 1967 (MEXU). Sin municipio especificado: Barranca of Guadalajara, hwy 41, $8 \mathrm{~km} \mathrm{~W}$ and S of Puente Guadalupe, 15 septiembre 1983, W. R. Anderson 12655 (ENCB, MICH, NY); Jalisco, junio-octubre 1886, E. Palmer 346 (NY, US); upper edge on marsh meadow by road to Tapalpa $12 \mathrm{mi}$, W of jct. with road to Colima, 11 agosto 1966, R. Kral 27584 (ENCB); SW de San Juan de los Lagos, 12 agosto 1992; A. Le Duc et al. 256 (MEXU); 4 miles east of Tapalpa, 3 noviembre 1960, R. McVaugh 20711 (MICH); sin localidad, agosto 1886. E. Palmer $s / n$ (GH). Michoacán: Mpio. Charo: Cañon de la caída del Salto, $20 \mathrm{~km}$ E of Morelia, 25 julio 1966, F. Barkley \& D. J. Carr 36116 (GH). Mpio. Ciudad Hidalgo (Hidalgo): hyw. 15, $7 \mathrm{~km} \mathrm{~W}$ of Ciudad Hidalgo, 29 junio 1964, G. Mick \& K. Roe 167 (ENCB); carretera Ciudad Hidalgo - Morelia, Puerto los Tepetates, 23 agosto 1997, E. Solano \& C. Correa 1133 (FEZA, MEXU); $2 \mathrm{~km}$ adelante del Puerto los Tepetates, sobre la carretera Ciudad Hidalgo - Morelia, 23 agosto 1997, E. Solano \& C. Correa 1134 (FEZA). Mpio. Coeneo: $3 \mathrm{~km}$ al sur de Constitución, 2 agosto 1988, P. Ramos 163. (IEB, MEXU, XAL); Coeneo, sin localidad, 26 julio 1991, J. M. Escobedo 1987 (IEB, MEXU). Mpio. Erongarícuaro: $3 \mathrm{~km}$ antes de llegar a Zinciro, 17 julio 1995, E. Solano et al. 798 (FEZA). Mpio. Lagunillas: La Caja, 4 julio 1986, J. M. Escobedo 975 (CHAP, IEB, XAL); 9 km sobre la desviación a Lagunillas en el camino Morelia - Quiroga, 9 agosto 1991, M. Flores et al. 574 (UAMIZ). Mpio. Maravatío: 0.5 km antes de la desviación a Santiaguito, 2 km antes de Santa Mónica, rumbo a Maravatío, 10 agosto 1991, A. Espejo et al. 4571 (IEB); carretera 126, libre a Morelia, a la altura del $\mathrm{km}$ 95, aproximadamente $30 \mathrm{~m}$ al E de la carretera, SE de Santa Mónica, 8 septiembre 1994, E. Solano et al. 766 (FEZA); a la altura del km 95, sobre la carretera libre de Maravatío a Morelia, antes de llegar a Santa Mónica, 16 julio 1995, E. Solano et al. 796 A (FEZA). Mpio. Morelia: cerro Azul, 11 septiembre 1909, G. Arsène (GH, US); sin localidad, 4 julio 1909, G. Arsène 1910 (MEXU); Cerro Azul, vicinity of 
Morelia, 1910, G. Arsène 6762 (US); vicinity of Morelia, monte San Miguel, agosto 1911, G. Arsène (US); camino a Cerro del Águila, 11 agosto 1991, E. García \& M. Sánchez 3769 (IEB, MEXU); in pascuis Morelia, 1840, L. Hartweg 405 (NY); cerca de San Miguel del Monte al S de Morelia, 18 agosto 1938, L. A. Knoyer A524 (MICH); Agua Zarca, SO de San Miguel del Monte, 27 agosto 1989, C. Medina 889 (IEB); 4 km al S de Jesús del Monte, 20 julio 1986, J. Rzedowski 39338 (ENCB, IEB); cerca del puerto de los Copales, $8 \mathrm{~km}$ al E de Morelia, sobre la carretera a Mil Cumbres, 17 julio 1986, J. Rzedowski 39866 (IEB, ENCB); vertiente norte del Pico Azul, cerca de San José de las Torres, 7 diciembre 1986, J. Rzedowski 41265 (IEB); camino del rancho Río Bello al Cerro Pico Azul, 15 septiembre 1987, J. S. Martínez 2209 (IEB). Mpio. Paracho: $300 \mathrm{~m}$ al N de Aranza, salida a Cheranastico, 28 julio 1987, M. Pérez 114 (ENCB, IEB, MEXU, XAL); $500 \mathrm{~m}$ al SW de Aranza, sobre el camino de terracería que va a Cheranastico, 10 septiembre 1994, E. Solano \& C. Correa 775 (FEZA). Mpio. Puruándiro: $9 \mathrm{~km}$ al SE de Puruándiro, sobre la carretera a Cuitzeo, 22 junio 1985, H. Díaz 1119 (IEB). Mpio. Queréndaro: casi $2 \mathrm{~km}$ después del río Parras, sobre la terracería Queréndaro - Real de Otzumatlán, 23 julio 1998, A. R. López et al. (UAMIZ). Mpio. Tizapotla (?): camino del Zapote a Cerro Frío, 2 noviembre 1989, E. Espejo et al. 3861. (UAMIZ). Mpio. Villa Jiménez: Hoyaila en la Alberca, 28 julio 1983, J. N. Labat 313 (IEB, MEXU); Cerro el Brinco, 16 agosto 1990, E. Pérez \& E. García 1538 (IEB). Mpio. Villa Morelos (Morelos): más o menos $3 \mathrm{~km}$ al WNW de Villa Morelos, 21 julio 1998, E. Carranza 5555 (MEXU). Mpio. Zacapu: carretera 15 Zacapu - Zamora a la altura del km 102, E. Solano et al. 801 (FEZA, MEXU), Malpaís Negro, más o menos $7 \mathrm{~km}$ al SW de los Espinos, 26 julio 1988, S. Zamudio \& A. Grimaldo 6668 (IEB, MEXU). Mpio. Zamora: aproximadamente $3 \mathrm{~km}$ después del entronque Zacapu - Zamora, yendo hacia Zacapu, sobre la carretera 15, 9 agosto 1996, E. Solano \& C. Correa 864 (FEZA). Mpio. Zinapécuaro: La Lagunita, $2.5 \mathrm{~km}$ al S de Cruz de Caminos, 4 agosto 1989, M. J. Jasso 1335 (IEB, MEXU); El Zapote, sobre la carretera 51, a la altura del km 24 viniendo de Ciudad Hidalgo, $1 \mathrm{~km}$ al E del Zapote, 8 septiembre 1994, E. Solano et al. 773 (FEZA, MEXU); carretera 161, casi $10 \mathrm{~km}$ de la desviación a Ucareo, yendo hacia Morelia, 16 julio 1995, E. Solano et al. 797 (FEZA, MEXU). Sin municipio especificado: sin localidad, 4 julio 1909, G. Arsène 2184 (MEXU); road 15 between km 235 and 236 nearly $24 \mathrm{~km} \mathrm{~W}$ of Ciudad Hidalgo, 6 julio 1968, $R$. W. Cruden 1340 (INIF); casi $15 \mathrm{~km}$ de Morelia, 9 julio 1959, The College of Idaho Field Biology Expedition s/n (MEXU); km 9-17 road to Pátzcuaro, from highway 15, 22 julio 1971, A. C. Gibson \& L. C. Gibson 2314 (ENCB, RSA); colonia Lázaro Cárdenas, cerca de Tzintzuntzan, 6 agosto 1985, J. Rzedowski 38897 (IEB); Cerro de 
Guadalupe, al E de Acuitzio del Canje, 24 julio 1986, H. Díaz 2475A (ENCB, IEB, MEXU). Morelos: Mpio. Puente de Ixtla: después de la Tigra rumbo a el Zapote, A. Espejo et al. 5679 (UAMIZ); camino del Zapote a Cerro Frío, 2 noviembre 1989, A. Espejo et al. 3861 (UAMIZ). NAYARIT: Sin municipio especificado: about $10 \mathrm{mi}$ SE of Ahuacatlán along road to Barranca del Oro, 26 agosto 1971, W. D. Stevens 1459 (ENCB, MICH). Querétaro: Mpio. Amealco de Bonfil: $18.5 \mathrm{~km}$ después de San Juan del Río rumbo a Amealco, 22 julio 1998, J. Ceja 661 (UAMIZ). Sin municipio especificado: camino entre carretera a México y Amealco, más o menos a la mitad, 9 agosto 1976, E. Argüelles 480 (ENCB, MEXU). Tlaxcala: Mpio. Españita: el Potrero, $10 \mathrm{~km}$ al SE de Españita, 15 agosto 1993, R. I. Ramírez et al. 502 (FEZA); carretera Calpulalpan - Españita, $1.2 \mathrm{~km}$ antes de la desviación a Españita, $100 \mathrm{~m}$ al E de la carretera, 4 septiembre 1994, E. Solano et al. 764 (FEZA, MEXU); carretera Calpulalpan - Tlaxcala, $3 \mathrm{~km}$ al E de Españita, $200 \mathrm{~m}$ al E de la carretera, 4 septiembre 1994, E. Solano et al. 765 (FEZA, MEXU); $500 \mathrm{~m}$ antes de llegar al entronque a Españita, sobre la carretera Calpulalpan - Veracruz, 11 julio 1996, E. Solano et al. 844 (FEZA, MEXU); carretera desviación a Apizaco, casi 500 $\mathrm{m}$ al S del Potrero Grande, 11 julio 1996, E. Solano et al. 846 (FEZA, MEXU). Mpio. Nanacamilpa: $5 \mathrm{~km}$ antes de la desviación a Españita, siguiendo la carretera Texcoco - Tlaxcala, 8 julio 1995, E. Solano et al. 790 (FEZA). Sin municipio especificado: sin localidad, únicamente México, 4 agosto 1904, C. G. Pringle 13221 (US); sin localidad, únicamente México, 11 agosto 1885, W. Schumann 1475 (US).

Polianthes geminiflora (Lex.) Rose var. pueblensis E. Solano \& García-Mend., Acta Bot. Mex. 78: 111-123. 2007. TIPO: MÉXICO: Puebla, $4 \mathrm{~km}$ al norte de la presa Manuel Ávila Camacho (Valsequillo), 12 julio 1996, E. Solano et al. 849 (holotipo: MEXU!; isotipos: CHAPA!, ENCB!, FEZA!, HUAP!, HUMO!, IEB!, US!).

Hierbas perennes. Hojas 3-6 por roseta, 14-17 $\mathrm{cm}$ de largo, 1-2 $\mathrm{mm}$ de ancho, lineares, ápice agudo, margen regularmente denticulado. Inflorescencia racemosa, 35-65 cm de largo; primera bráctea estéril, 11.7-15 cm de largo, 1-2 mm de ancho, lineares a lanceoladas, ápice agudo o acuminado, denticulado. Flores con el tubo periantal 15-21 mm de largo, 1.5-3.0 mm de ancho por debajo de los lóbulos, lóbulos 2.0-2.5 mm de largo, 1-2 mm de ancho; estambres con los filamentos de 1.0-1.7 cm de largo, insertados 3-5 mm por arriba del ápice del ovario; anteras 5-6 $\mathrm{mm}$ de largo. Fruto 1.2-1.5(-1.7) cm de largo, 0.9-1.1 cm de ancho.

Fenología. Florece de julio a agosto y fructifica de septiembre a noviembre. 
Hábitat y distribución. Se encuentra en áreas abiertas de bosque de encino, bosque de Juniperus, matorral xerófilo, pastizal y campos de cultivo de maíz. En suelos calcáreos, de color gris o negro; con textura arenosa, arcillosa o migajón arcillosa; en poblaciones muy localizadas con pocos individuos. En altitudes de 2160 a $2220 \mathrm{~m}$.

Nombre común. Arete, aretito, coral.

Ejemplares examinados: Puebla: Mpio. Amozoc: Chachapa al cerro de la derecha, 10 agosto 1986, M. Rodríguez 342 (HUAP). Mpio. Cuauhtinchán: Cerro Xonaca al norte de Cuauhtinchán, s/f de recolecta, Olivas-Lazcano 895 (HUAP); carretera 528, $3 \mathrm{~km}$ antes de llegar a Cuauhtinchán, ejido San Pedro Alpatlahua, 25 julio 1996, E. Solano \& Ma. del C. López 853 (FEZA, MEXU). Mpio. Chila: $1 \mathrm{~km}$ al sur de Yucunduchi, por la carretera a Huajuapan, 10 julio 2001, A. García M. et al. 7113 (MEXU). Mpio. Puebla: Africam Safari en el valle de Valsequillo, 1 agosto 1994, M. Rodríguez 895 (HUAP); sobre la carretera a Africam Safari, enfrente del Zoológico, 9 agosto 1995, E. Solano et al. 794 (FEZA, MEXU); alrededores de la colonia Patria Nueva, 12 julio 1996, E. Solano \& C. Correa 847 (FEZA, MEXU); $2 \mathrm{~km}$ del entronque a Africam Safari, 12 julio 1996, E. Solano \& C. Correa 848 (FEZA, MEXU); carretera Puebla - Azomiatla aproximadamente $2 \mathrm{~km}$ viniendo de Azomiatla, 13 julio 1996, E. Solano \& C. Correa 852 (FEZA, MEXU). Mpio. San Baltazar Tetela (Tetela de Ocampo): $5 \mathrm{~km}$ al oriente de San Baltazar Tetela, 13 julio 1996, E. Solano et al. 850 (FEZA, MEXU); entre la Libertad y la Paz Tlaxcolpa, carretera a Azomiatla, 13 julio 1996, E. Solano \& C. Correa 851 (FEZA, MEXU). Mpio. Tepenene: 4 km al NE de la Presa Miguel Ávila Camacho, 12 julio 1996. E. Solano \& C. Correa 849. (FEZA, MEXU). Mpio. Valsequillo: Africam Safari, $M$. Rodríguez 895 (HUAP). Sin localidad exacta ni fecha de recolecta, C. Ehrenberg 259 (MEXU).

\section{AGRADECIMIENTOS}

A Carlos Correa D., por su compañía durante las recolectas en el área de distribución del género. Raquel Galván, Sergio Zamudio y dos revisores anónimos hicieron importantes observaciones que mejoraron el escrito final. Magdalena Ayala H. Preparó el mapa de distribución geográfica y Jesús Salgado V. Realizó la ilustración y tomó la fotografía que se incluye en el trabajo. 


\section{LITERATURA CITADA}

Espejo-Serna, A., A. R. López-Ferrari y M. Flores-Cruz. 1993. Neotipificación de Pitcairnia vallisoletana Lex. (Bromeliaceae). Acta Bot. Mex. 23: 53-58.

García-Mendoza, A. y E. Solano. 2007. Polianthes oaxacana y P. geminiflora var. pueblensis (Agavaceae), taxa nuevos de México. Acta Bot. Mex. 78: 111-123.

González, B. A. 1998. Descripción morfológica y anatómica del tallo de Polianthes L. (Agavacaeae). Tesis de Licenciatura. Facultad de Estudios Superiores Zaragoza, Universidad Nacional Autónoma de México. México, D.F., México. 37 pp.

Linneaus, C. 1738. Hortus Cliffortianus. Amsterdam. p. 126.

Llave, P. de la e I. Lexarza. 1824. Novorum vegetabilium descriptiones. Fasciculus 1. Apud Martinum Riveram. Mexici. p. 6.

McVaugh, R. 1989. Polianthes L. In: Anderson, W. R. (ed.). Flora Novo-Galiciana 15: 247260.

Rose, J. N. 1903. Studies of Mexican and Central American plants. Contr. U. S. Natl. Herb. 8: $1-55$.

Solano, C. E. 2000. Sistemática del género Polianthes L. Agavacaeae. Tesis doctoral. Facultad de Ciencias, Universidad Nacional Autónoma de México. México, D.F., México. $291 \mathrm{pp}$.

Stafleu, F. A. y R. S. Cowan. 1979. Taxonomic literature. A selective guide to botanical publications and collections with dates, commentaries and types. 2a. ed. Vol. 2. Bohn, Scheltema \& Holkema. Utrecht, The Netherlands. 991 pp. 